\title{
La gestión de la calidad en Perú: un estudio de la norma ISO 9001, sus beneficios y los principales cambios en la versión $2015^{*}$
}

\section{Quality Management in Peru: A Study of ISO 9001 Standard, its Benefits and the Main Changes in the 2015 Version}

\section{A gestão da qualidade no Peru: um estudo da norma ISO 9001, seus benefícios e as principais mudanças na versão 2015}

\author{
Edmundo R. Lizarzaburu Bolaños ${ }^{\star \star}$
}

Fecha de recibido: 9 de febrero de 2015

Fecha de aprobado: 25 de agosto de 2015

Doi: dx.doi.org/10.12804/rev.univ.empresa.30.2016.02

Para citar este artículo: Lizarzaburu Bolaños, E. R. (2016). La gestión de la calidad en Perú: un estudio de la norma ISO 9001 , sus beneficios y los principales cambios en la versión 2015. Universidad \& Empresa, 18(30), 33-54. Doi: dx.doi.org/10.12804/rev.univ.empresa.30.2016.02

\section{RESUMEN}

Debido a la gran relevancia que tiene la gestión de calidad en la actual coyuntura internacional, el presente trabajo de investigación revisa y analiza la más destacada norma en esta materia: la ISO 9001. En el documento, se establecen los orígenes y la evolución del término

* Este documento fue elaborado con la colaboración de Fiorella Castillo, estudiante de la Universidad ESAN (Lima, Perú).

** Doctorando en Investigación de Ciencias de la Administración, de la Universidad Carlos III (España), Global MBA de la Thunderbird School of Global Management (Estados Unidos) y la Escuela de Graduados en Administración y Dirección de Empresas (Egade) del Tecnológico de Monterrey (México), magíster en Contabilidad y Finanzas, de la Universidad de San Martín de Porres (Perú), ingeniero industrial de la Pontificia Universidad Católica del Perú. Profesor del Departamento de Finanzas de la Universidad ESAN (Perú). Correo electrónico: elizarzaburu@esan.edu.pe 
calidad. Este se define mediante la descripción de la familia de normas. Se identifican también su estructura y los principios sobre los que se basa. A partir de lo anterior, como un resultado adicional del trabajo, se detallan los beneficios cualitativos y cuantitativos que la norma brinda a las empresas que se adscriben a la misma. Por último, se establecen las principales modificaciones que incorporara la nueva ISO 9001 versión 2015.

Palabras clave: calidad, certificación de calidad, gestión de la calidad, ISO 9001:2015.

\section{ABSTRACT}

Considering the great importance of quality management at the present international juncture, this research reviews and analyzes the most important standard pertaining thereto, i.e. ISO 9001. In this paper, the origins of the term "quality" are established, as well as its evolution over time. It is defined by using the description of the family of standards. In addition, its structure and also the principles on which it is based were identified. Following the above and as an additional result of this work, the qualitative and quantitative benefits with which the standard provides companies registered with it are detailed. Finally, the main changes that the new ISO 9001:2015 revision will include are established.

Keywords: Quality, quality certification, quality management, ISO 9001:2015.

\section{RESUMO}

Considerando a grande relevância que tem a gestão da qualidade na atual a conjuntura internacional, o presente trabalho de investigação faz uma revisão e uma análise da mais destacada norma nesta matéria: ISO 9001. No documento se estabelecem as origens do termo qualidade assim como a evolução que o mesmo tem tido ao longo do tempo. Este define-se fazendo uso da descrição da família de normas. Se identifica também a sua estrutura assim como os princípios nos que se baseia. A partir do anterior, como um resultado adicional do trabalho, detalham-se os benefícios qualitativos e quantitativos que a norma brinda às empresas que se adscrevem à mesma. Finalmente, estabelecem-se as principais modificações que a nova ISO 9001 versão 2015 incorporará.

Palavras-chave: qualidade, certificação de qualidade, gestão da qualidade, ISO 9001:2015. 


\section{INTRODUCCIÓN}

En la actualidad, el mercado se modifica a pasos agigantados. Hace algunos años, las empresas comercializaban sus productos y servicios en el escenario local. Esto, de algún modo, les permitía mantener cierto grado de competitividad - suficiente para mantenerse $y$, en no pocos casos, para crecer y desarrollarse- . Sin embargo, hoy esta situación ha cambiado.

En el presente, las compañías de múltiples países, tanto de Naciones desarrolladas como de economías emergentes - tal es el caso de Perú - han logrado abrirse al mercado exterior. Estas han conseguido extender sus negocios y darles un mayor alcance, aunque el número de empresas que en este país lo ha logrado es aún muy reducido. En un principio, la razón principal estaba en las barreras existentes al libre comercio, pero hoy esta no es una causa relevante.

Perú cuenta con diecinueve tratados de libre comercio (TLC) vigentes, cuyo objetivo principal es beneficiar, en cuanto sea posible, las exportaciones del país, por medio de la eliminación de las barreras al intercambio. Con estas iniciativas, se busca que millones de consumidores en el mundo puedan conocer y acceder a los productos y servicios elaborados en esta Nación (Ministerio de Comercio Exterior y Turismo, 2014).

Los acuerdos firmados por Perú eliminan una gran cantidad de barreras al comercio, como una prueba de que esta ya no es la razón básica de la reducida comercialización de ese país con el exterior. El problema ahora radica en que los negocios locales no cuentan con la preparación necesaria y las condiciones requeridas para competir de manera adecuada en el mercado internacional. Una parte importante de estas condiciones tiene que ver con el mejoramiento de su oferta de productos y servicios, para que cuenten con una calidad como la exigida en el escenario global. Esto, entre otras medidas relevantes, mediante la obtención de reconocidas certificaciones como la atinente al cumplimiento de la norma ISO 9001.

Una prueba de lo anterior es que "Perú es uno de los países con menor número de empresas certificadas en gestión de calidad" (Instituto nacional de defensa de la competencia y de la protección de la propiedad intelectual [Indecopi], 2012a). En 2013, el país contaba con alrededor de 1.200 empresascertificadasISO9001(Centro de Desarrollo Industrial [CDI], 2013), solo doscientas más que el 
año anterior (Indecopi, 2012a). Esto significa que de 1.713.272 empresas existentes (Instituto Nacional de Estadística e Informática [INEI], 2013), solo un $0,071 \%$ estaba certificado.

Las empresas peruanas ya no tienen que lidiar con la serie de barreras al comercio internacional que antes enfrentaban. Ahora, se ven en la necesidad de mejorar sus procesos internos para ofrecer productos y servicios competitivos en el mercado mundial. Un medio básico para lograr esta mejora, como ya se ha indicado, es la obtención de reconocidas certificaciones de calidad como la ISO 9000. Para las empresas, "es importante tener una certificación [...] pues así puede demostrar a su cliente, sea consumidor local o extranjero, que puede producir siempre con la misma calidad" (Indecopi, 2012a).

Este es un aspecto importante en el contexto global, porque "está demostrado que aquellas empresas que cuentan con normas de calidad en sus procesos productivos mejoran su desempeño, tanto en el mercado local como internacional, mostrando un incremento en sus ventas y exportaciones" (Indecopi, 2012b).

Debido a la gran relevancia de la norma ISO 9000 (Hoyle, 2006), en el presente trabajo se estudian y ana- lizan sus antecedentes, su desarrollo y revisión más recientes, es decir, la prevista para el segundo semestre de 2015. Para esto, en primer lugar, se contextualiza la norma desde la literatura pertinente; luego, se observan algunos de los aspectos más relevantes de la versión 2008; después, se analizan los principales beneficios de la norma; más adelante, se identifican los cambios de la nueva versión (ISO 9001:2015) y, por último, se presentan las principales conclusiones del trabajo realizado.

\section{REVISIÓN DE LA LITERATURA \\ 1.1. Calidad}

Para comenzar con el análisis de la norma ISO 9001 conviene analizar el concepto de calidad con el cual se vincula. La calidad tiene que ver con cuán adecuado es un producto o servicio para el uso que se pretende hacer de él; en otras palabras, para aquello que desea el cliente. Implica tratar de satisfacer las necesidades de los consumidores $\mathrm{y}$, en la medida de lo posible, superar sus expectativas (Alcalde San Miguel, 2008).

De acuerdo con Cuatrecasas (2012), la calidad es "el conjunto de características que posee un producto o servicio obtenidos en un sistema productivo, así como la capacidad de satisfacción de los requerimientos 
del usuario" (p. 575). Tiene que ver con "las características provenientes de mercadeo, ingeniería, manufactura y mantenimiento que estén relacionadas directamente con las necesidades del cliente" (Feigenbaum, 1983, p. 7).

En relación con la norma ISO 9001, centrada en el concepto de calidad, se ha previsto la publicación de su quinta edición para el mes de septiembre de 2015. En el momento de elaboración del presente artículo está en proceso de preparación, con el fin de adecuar su contenido y alcance a la evolución del mercado y de las empresas.

De este modo, los principios de gestión de la calidad están siendo revisados, entre ellos, el relativo a la "participación de personas", el cual ha sido redefinido con la siguiente declaración: "Es esencial para la organización que todas las personas sean competentes, estén capacitadas y comprometidas con la generación de valor. La organización así, mejora su capacidad para generar valor a través de personas competentes, capacitadas y comprometidas" (International Organization for Standardization [ISO], 2013).

\subsection{Evolución de la calidad}

El concepto de calidad ha evolucionado bastante desde sus orígenes. Este proceso ha permitido aumentar y refinar sus objetivos y lograr que se enfoque en la satisfacción plena de las expectativas de los consumidores de bienes y los usuarios de servicios. En la figura 1 se incorpora una representación gráfica de este desarrollo durante los últimos años.

Figura 1. Evolución del concepto de calidad

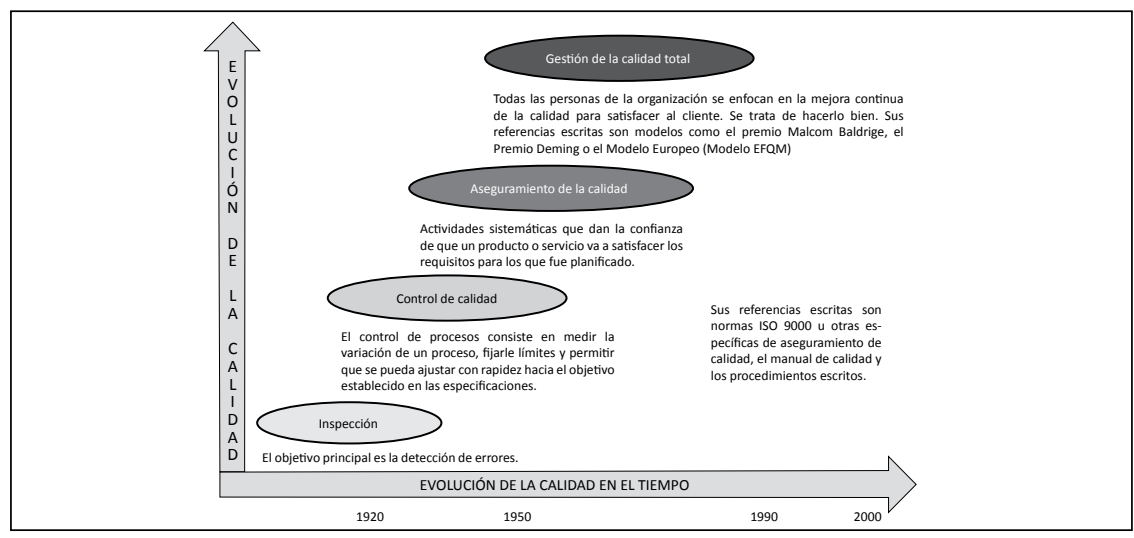

Fuente: Alcalde San Miguel (2008, p. 25). 


\subsection{La International Organization for Standardization (ISO)}

Para abordar la norma ISO 9001 es necesario recordar qué es la ISO. La Oficina Internacional del Trabajo ([OIT], 2007) define a la Organización Internacional de Normalización (ISO) de la siguiente manera:

La ISO es una organización que agrupa institutos nacionales de normalización a fin de promover en todo el mundo la elaboración de normas relacionadas con productos y servicios en sectores e industrias particulares. La ISO no es una organización internacional pública; sus miembros no son delegaciones de gobiernos nacionales. Muchos de los institutos de normalización forman parte de la estructura gubernamental de sus países o se rigen por mandatos del gobierno. Otros pertenecen al sector privado y han sido establecidos mediante alianzas de asociaciones de la industria (p. 1).

Esta institución sobresale en el actual sistema socioeconómico global. Se ha ocupado de señalar estándares en muy diversas áreas, no solo vinculadas con la calidad, sino también con temas como el ambiente, la tecnología de la información y un sinnúmero de asuntos adicionales relacionados con producción, comercialización y distribución de bienes y servicios.

\subsection{La familia de normas ISO 9000}

La ISO 9000 no es, stricto sensu, una norma, sino una familia de normas. El Comité Técnico ISO/TC 176 (2005, p. 6) da cuenta de sus cuatro miembros básicos, que describe de la siguiente manera:

- Norma ISO 9000: describe los fundamentos de los sistemas de gestión de la calidad y especifica su terminología.

- Norma ISO 9001: detalla los requisitos para los sistemas de gestión de la calidad, aplicables a toda organización que necesite demostrar su capacidad para proveer productos que cumplan los requerimientos de sus clientes y los aspectos reglamentarios aplicables. El objetivo básico es aumentar la satisfacción del consumidor.

- Norma ISO 9004: proporciona directrices que consideran tanto la eficacia como la eficiencia del sistema de gestión de la calidad. El objetivo de esta norma es la mejora del desempeño de la organización y la satisfacción de los clientes y de otras partes interesadas.

- Norma ISO 19011: brinda orientación relativa a las auditorías de sistemas de gestión de la calidad y de gestión ambiental. 
Estas cuatro normas que conforman la familia ISO 9000 se representan en la figura 2.

Figura 2. Familia de normas ISO 9000

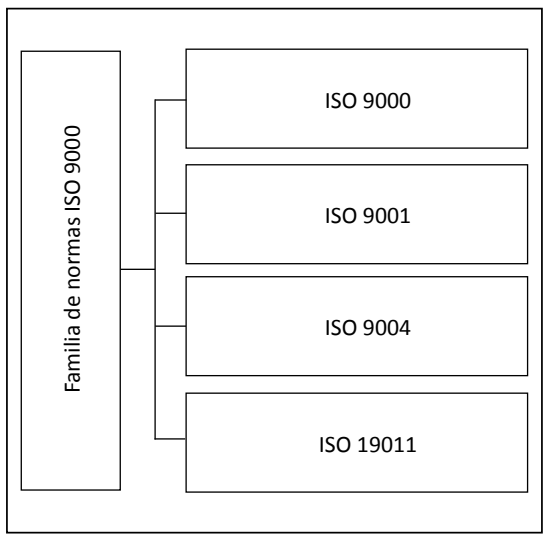

Fuente: Comité Técnico ISO/TC 176 (2005, pp. 6-7).

La ISO 9001 cuenta con gran relevancia. Es reconocida en el ámbito internacional y es considerada como el fundamento del sistema de gestión de la calidad y de la mejora. A continuación, se revisan sus principales elementos.

\section{LA NORMA ISO 9001:2008}

El Comité Técnico ISO/TC 176 (2008) sostiene que la norma ISO 9001:2008 puntualiza los requisitos para un sistema de gestión de la calidad, en particular en dos casos, cuando una organización:

1. Necesita demostrar su capacidad para proporcionar regularmente productos que satisfagan los requisitos del cliente, los legales y reglamentarios aplicables.

2. Aspira a aumentar la satisfacción del cliente a través de la aplicación eficaz del sistema (Comité Técnico ISO/TC 176, 2008).

Esta norma considera diversos elementos de carácter filosófico y conceptual, asociados con el discurso de la calidad y se materializan en una serie de principios que se presenta a continuación.

\subsection{Principios de gestión de la calidad}

La norma se basa en ocho principios de gestión de la calidad. El Comité Técnico ISO/TC 176 (2008) los define de la siguiente manera:

1. Enfoque al cliente: las organizaciones dependen de sus clientes; por lo tanto, deben comprender sus necesidades actuales y futuras, satisfacer sus requisitos y esforzarse por exceder sus expectativas.

2. Liderazgo: los líderes establecen la unidad de propósito y la orientación de la organización. Estos deben crear y mantener un ambiente interno en el cual el personal pueda involucrarse por completo en el logro de los objetivos de la empresa. 
3. Participación del personal: el personal constituye la esencia de una organización. Su compromiso total posibilita que sus habilidades sean usadas para el beneficio de la empresa.

4. Enfoque basado en procesos: un resultado deseado se alcanza con más eficiencia cuando las actividades y los recursos relacionados se gestionan como un proceso.

5. Enfoques del sistema para la gestión: identificar, entender y gestionar los procesos interrelacionados como un sistema contribuye a la eficacia y eficiencia de una organización en el logro de sus objetivos.
6. Mejora continua: la mejora continua del desempeño global de la organización debe ser su objetivo permanente.

7. Enfoque basado en hechos para la toma de decisiones: las decisiones eficaces se basan en el análisis de datos e información.

8. Relaciones beneficiosas con el proveedor: una organización y sus proveedores son interdependientes. Una relación mutuamente beneficiosa aumenta la capacidad de ambos para crear valor.

Estos principios, que proveen un fundamento filosófico esencial para la norma, se materializan en ocho puntos que se representan en la figura 3.

Figura 3. Principios de gestión de calidad

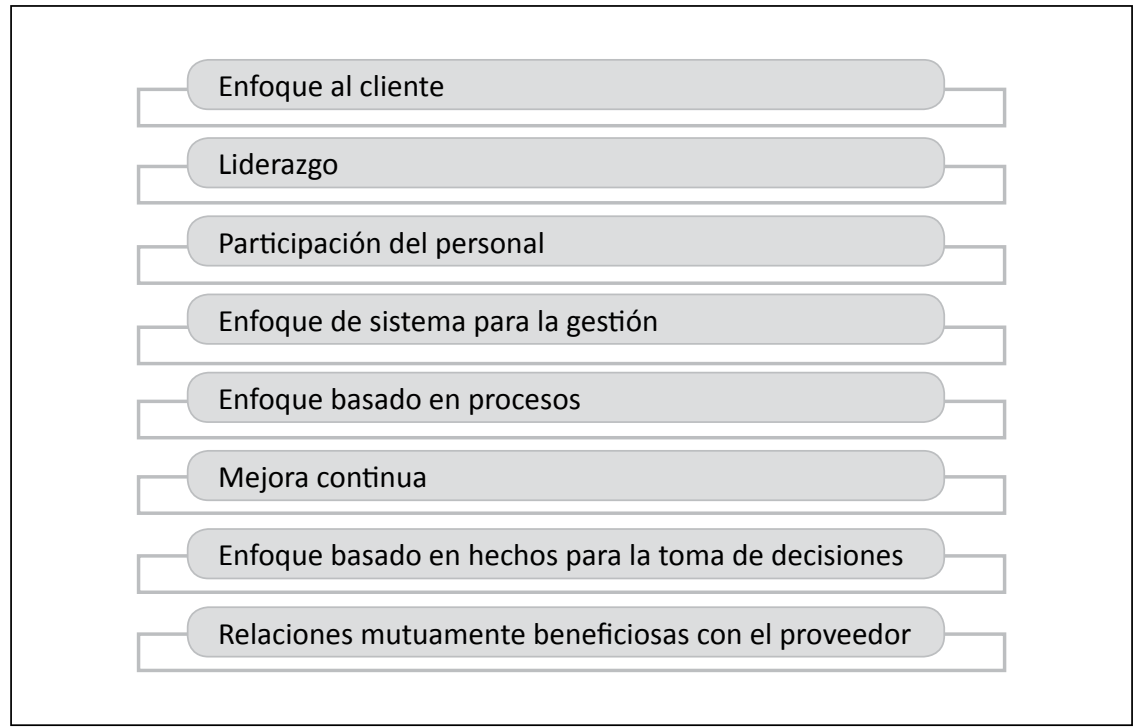

Fuente: Comité Técnico ISO/TC 176 (2005, pp. Vi-Vii). 
2.2. Estructura de la norma ISO 9001

La norma ISO 9001 está organizada en ocho secciones que se presentan en la figura 4.

\section{Figura 4. Estructura norma ISO 9001}

\begin{tabular}{|l|l|}
\hline 1 & Alcance, objetivo y campo de aplicación. \\
\hline 2 & Aspectos legales (normatividad). \\
\hline 3 & Terminología-definiciones. \\
\hline 4 & $\begin{array}{l}\text { Sistema de gestión de calidad-documenta- } \\
\text { ción }(4.1,4.2) .\end{array}$ \\
\hline 5 & $\begin{array}{l}\text { Responsabilidad de la dirección } \\
(5.1 ; 5.2,5.3,5.4,5.5,5.6) .\end{array}$ \\
\hline 6 & Gestión de recursos $(6.1,6.2,6.3,6.4)$. \\
\hline 7 & $\begin{array}{l}\text { Realización del producto } \\
(7.1,7.2,7.3,7.4,7.5,7.6) .\end{array}$ \\
\hline 8 & $\begin{array}{l}\text { Medición, análisis y mejora } \\
(8.1,8.2,8.3,8.4,8.5) .\end{array}$ \\
\hline
\end{tabular}

Fuente: elaboración propia con base en Comité Técnico ISO/TC 176 (2008).
Las cuatro primeras secciones identifican el ámbito, las definiciones y los términos para la norma; las cinco restantes contienen los requisitos para la implementación del sistema de gestión de calidad (figura 5).

A continuación, se ofrece una síntesis de los principales elementos que estructuran la norma - sin considerar el punto cero (introducción), porque es muy básico y tiene un carácter general- y una breve explicación de cada uno.

\subsubsection{Objeto y campo de aplicación}

El Comité Técnico ISO/TC 176 (2008) explica este aspecto de la siguiente manera:

Figura 5. Sistema de gestión de la calidad

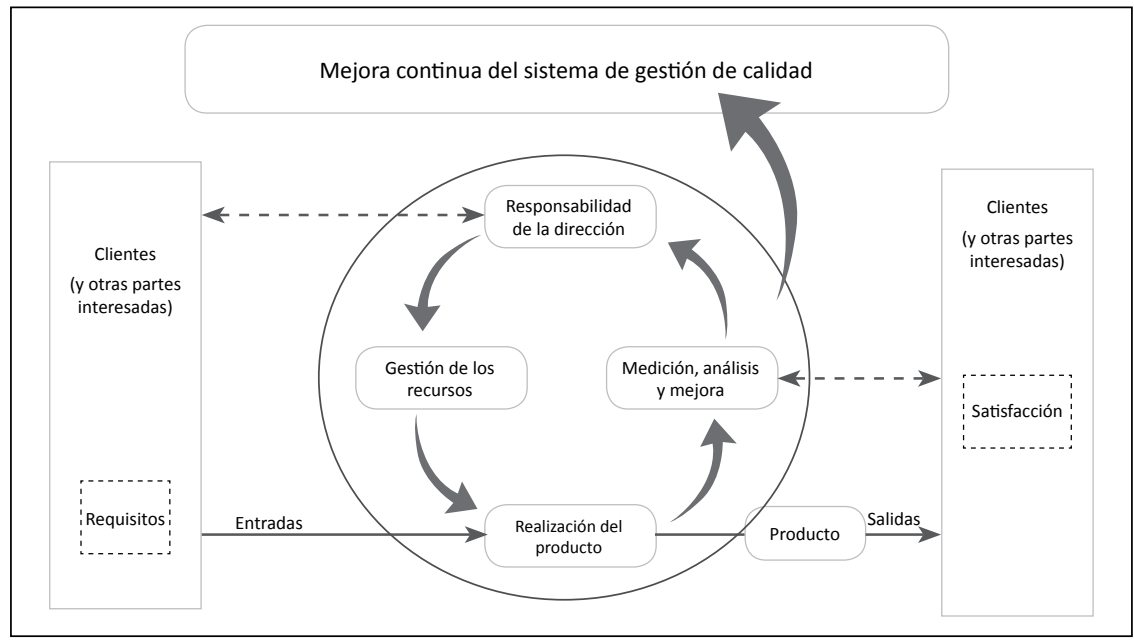

Fuente: Comité Técnico ISO/TC 176 (2005, p. 3). 
Esta Norma Internacional especifica los requisitos para un sistema de gestión de la calidad, cuando una organización:

Necesita demostrar su capacidad para proporcionar regularmente productos que satisfagan los requisitos del cliente, los legales y reglamentarios aplicables.

Aspira a aumentar la satisfacción del cliente a través de la aplicación eficaz del sistema.

Todos los requisitos de esta Norma Internacional son genéricos y se pretende que sean aplicables a todas las organizaciones sin importar su tipo, tamaño y producto suministrado (p.1).

\subsubsection{Referencia normativa}

El Comité Técnico ISO/TC 1760 (2008) define a la norma ISO 9000:2005, "Sistemas de gestión de la calidad. Fundamentos y vocabulario", como el documento de referencia en esta materia.

\subsubsection{Términos y definiciones}

El Comité Técnico ISO/TC 1760 (2008) aclara que: "A lo largo del texto de esta Norma Internacional, cuando se utilice el término 'producto', este puede significar también 'servicio"' (p. 2).

\subsubsection{Sistemas de gestión de calidad}

El Comité Técnico ISO/TC 176 (2008) explica este aspecto de la siguiente manera:

La organización debe establecer, documentar, implementar y mantener un sistema de gestión de la calidad y mejorar continuamente su eficacia de acuerdo con los requisitos de esta Norma Internacional. Para ello la organización debe:

- Determinar los procesos necesarios para el sistema de gestión de la calidad y su aplicación a través de la organización,

- Determinar la secuencia e interacción de estos procesos,

- Determinar los criterios y los métodos necesarios para asegurarse de que tanto la operación como el control de estos procesos sean eficaces,

- Asegurarse de la disponibilidad de recursos e información necesarios para apoyar la operación y el seguimiento de estos procesos,

- Realizar el seguimiento, la medición cuando sea aplicable y el análisis de estos procesos,

- Implementar las acciones necesarias para alcanzar los resultados planificados y la mejora continua de estos procesos (pp. 2-3).

En el contexto de la norma ISO 9001:2008, se especifican los requerimientos de documentación del 
sistema de gestión de calidad, que se muestran en la figura 6 .

Figura 6. Requisitos de la documentación

\begin{tabular}{|c|l|}
\hline 1 & Propósito de la empresa-adecuado. \\
\hline 2 & $\begin{array}{l}\text { Compromiso de cumplir los requisitos y } \\
\text { mejora continua. }\end{array}$ \\
\hline 3 & $\begin{array}{l}\text { Establecer un marco de referencia y } \\
\text { revisar objetivos de calidad. }\end{array}$ \\
\hline 4 & Comunicada y entendida en la empresa. \\
\hline 5 & Revisión continua. \\
\hline
\end{tabular}

Fuente: elaboración propia con base en Comité Técnico ISO/TC 176 (2008).

\subsubsection{Responsabilidad de la dirección}

El Comité Técnico ISO/TC 176 (2008) explica los siete elementos considerados en este aspecto de la siguiente manera:

a. Compromiso de la dirección: la Dirección debe proporcionar evidencia de su compromiso con el desarrollo y la implementación del sistema de gestión de la calidad, así como con la mejora continua de su eficacia (figura 7).

\section{Figura 7. Proceso de mejora de la eficacia}

\begin{tabular}{|c|l|}
\hline 1 & Política de calidad \\
\hline 2 & Manual de calidad \\
\hline 3 & Procedimientos \\
\hline 4 & Documentación \\
\hline 5 & Registros \\
\hline
\end{tabular}

Fuente: elaboración propia con base en Comité Técnico ISO/TC 176 (2008). b. Enfoque al cliente: la alta dirección debe asegurarse de que los requisitos del cliente se determinan y se cumplen con el propósito de aumentar la satisfacción del cliente. c. Enfoque de la calidad: dentro de la norma ISO 9001 se definen las características con las que una política de calidad debe contar (figura 8).

Figura 8. Características de la política de calidad

\begin{tabular}{|c|l|}
\hline 1 & $\begin{array}{l}\text { Satisfacer los requisitos del cliente y } \\
\text { legales }\end{array}$ \\
\hline 2 & Diseñar la política de calidad \\
\hline 3 & Determinar los objetivos de calidad \\
\hline 4 & Realizar la revisión por parte de la dirección \\
\hline 5 & Contar con los recursos \\
\hline
\end{tabular}

Fuente: elaboración propia con base en Comité Técnico ISO/TC 176 (2008).

d. Planificación: es importante indicar que la alta dirección debe asegurarse de que

- La planificación del sistema de gestión de la calidad debe realizarse teniendo como fin el cumplimiento de los requisitos, así como los objetivos de la calidad, y

- Se mantenga y asegure la integridad del sistema de gestión de la calidad cuando se realicen las etapas de planifican e implementan cambios en este.

e. Responsabilidad, autoridad y comunicación: [...] es importante que la alta dirección designe a un miembro de la dirección de la organización quien, independientemente de otras responsabilidades, debe ser el 
responsable y tener la autoridad que incluya:

- Asegurarse y garantizar que se establecen, implementan y mantienen los procesos necesarios para el sistema de gestión de la calidad,

- Mantener informado a la alta dirección sobre el desempeño del sistema de gestión de la calidad y de cualquier necesidad de mejora, y

- Promover la toma de conciencia (dentro de la empresa) de los requisitos del cliente a todo nivel.

f. Comunicación interna: la comunicación es un aspecto importante y por ello la alta dirección debe asegurarse de que se implementen los procesos de comunicación necesarios y adecuados dentro de la organización y de que la comunicación se realice tomando en consideración la eficacia del sistema de gestión de la calidad. g. Revisión por la dirección: es necesario y vital que la alta dirección revise de manera periódica el sistema de gestión de la calidad de la organización, a intervalos planificados, para asegurarse de su conveniencia, adecuación y eficacia continuas. La revisión debe incluir la evaluación de las oportunidades de mejora y la necesidad de efectuar cambios en el sistema de gestión de la calidad, incluyendo la política de la calidad y los objetivos de la calidad (pp. 4-6).

\subsubsection{Gestión de los recursos}

En la norma 9001: 2008 se define el objetivo por el cual las organizaciones deben proveerse de recursos, así como también los tres tipos de recursos que es necesario gestionar (figura 9).

\section{Figura 9. Gestión de recursos}

\begin{tabular}{|c|c|c|c|}
\hline Provisión de recursos & Recursos humanos & Infraestructura & Ambiente de trabajo \\
\hline $\begin{array}{l}\text { - Implementar } \\
\text { y mantener } \\
\text { el sistema de } \\
\text { gestión de la } \\
\text { calidad. } \\
\text { - Mejorar } \\
\text { continuamente su } \\
\text { eficacia. } \\
\text { - Aumentar la } \\
\text { satisfacción del } \\
\text { cliente mediante } \\
\text { el cumplimiento } \\
\text { de sus requisitos. }\end{array}$ & $\begin{array}{l}\text { - Determinar la competencia del personal } \\
\text { que realiza los trabajos que afectan a } \\
\text { la conformidad con los requisitos del } \\
\text { producto. } \\
\text { - Realizar las acciones necesarias para lograr } \\
\text { la competencia requerida. } \\
\text { - Evaluar la eficacia de las acciones tomadas. } \\
\text { - Asegurarse de que el personal sea } \\
\text { consciente de la pertinencia e importancia } \\
\text { de sus actividades y de cómo contribuyen } \\
\text { al logro de los objetivos de la calidad. } \\
\text { - Mantener los registros apropiados de } \\
\text { la educación, formación, habilidades y } \\
\text { experiencia. }\end{array}$ & $\begin{array}{l}\text { - La organización } \\
\text { debe determinar, } \\
\text { proporcionar } \\
\text { y mantener la } \\
\text { infraestructura } \\
\text { necesaria para lograr } \\
\text { la conformidad con } \\
\text { los requisitos del } \\
\text { producto. } \\
\text { - La infraestructura } \\
\text { incluye edificios, } \\
\text { espacio de trabajo, } \\
\text { equipos para los } \\
\text { procesos y servicios } \\
\text { de apoyo. }\end{array}$ & $\begin{array}{l}\text { - La organización } \\
\text { debe } \\
\text { determinar } \\
\text { y gestionar } \\
\text { el ambiente } \\
\text { de trabajo } \\
\text { necesario } \\
\text { para lograr la } \\
\text { conformidad } \\
\text { con los } \\
\text { requisitos del } \\
\text { producto. }\end{array}$ \\
\hline
\end{tabular}

Fuente: Comité Técnico ISO/TC 176 (2008, p. 8, 15). 


\subsubsection{Realización del producto}

El Comité Técnico ISO/TC 176 (2008) señala que la norma ISO 9001 divide este proceso en seis grupos:

\section{La planificación de la realización} de producto: incluye los elementos necesarios para la elaboración del producto tomando como base los requisitos del cliente.

2. Los procesos relacionados con el cliente: incluye todos los procesos involucrados para determinar los requisitos del producto hasta la comunicación con el cliente para la verificación de la información generada.

3. Diseño y desarrollo del producto: abarca desde la planificación para la elaboración hasta la revisión y verificación.

4. Las compras: la organización debe asegurarse de que el producto adquirido cumple con los requisitos de compra especificados. El tipo y el grado del control aplicado al proveedor y al producto adquirido deben depender del impacto de este último sobre la posterior realización del producto o sobre el resultado final.

5. Producción y prestación del servicio: este proceso está relacionado con el tipo de actividad de la empresa.

6. Control del seguimiento y de la medición: este proceso tiene que ver con las actividades de control de los indicadores establecidos.

\subsubsection{Medición, análisis y mejora}

El Comité Técnico ISO/TC176 (2008) considera cuatro elementos, de la siguiente manera:

\section{a. Seguimiento y medición}

\section{Satisfacción del cliente}

Como una de las medidas del desempeño del sistema de gestión de la calidad, la organización debe realizar el seguimiento de la información relativa a la percepción del cliente con respecto al cumplimiento de sus requisitos por parte de la organización.

Seguimiento y medición de los procesos

La organización debe aplicar métodos apropiados para el seguimiento, y cuando sea aplicable, la medición de los procesos del sistema de gestión de la calidad. Estos métodos deben demostrar la capacidad de los procesos para alcanzar los resultados planificados.

Cuando no se alcancen los resultados planificados, deben llevarse a cabo correcciones y acciones correctivas, según sea conveniente $[\ldots]$.

Seguimiento y medición del producto

La organización debe hacer el seguimiento y medir las características del 
producto para verificar que se cumplen los requisitos del mismo [...].

\section{b. Control del producto no con- forme}

La organización debe asegurarse de que el producto que no sea conforme con los requisitos del producto, se identifica y controla para prevenir su uso o entrega no intencionados. Se debe establecer un procedimiento documentado para definir los controles y las responsabilidades y autoridades relacionadas para tratar el producto no conforme.

\section{c. Análisis de datos}

La organización debe determinar, recopilar y analizar los datos apropiados para demostrar la idoneidad y la eficacia del sistema de gestión de la calidad y para evaluar dónde puede realizarse la mejora continua de la eficacia del sistema de gestión de la calidad.

Esto debe incluir los datos generados del resultado del seguimiento y medición y de cualesquiera otras fuentes pertinentes.

\section{d. Mejora}

\section{Mejora continua}

La organización debe mejorar continuamente la eficacia del sistema de gestión de la calidad, los objetivos de la calidad, los resultados de las auditorías, el análisis de datos, las acciones correctivas y preventivas y la revisión por la dirección.

\section{Acción correctiva}

La organización debe tomar acciones para eliminar las causas de las no conformidades con el objeto de prevenir que vuelvan a ocurrir. Las acciones correctivas deben ser apropiadas a los efectos de las no conformidades encontradas.

\section{Acción preventiva}

La organización debe determinar acciones para eliminar las causas de no conformidades potenciales para prevenir su ocurrencia. Las acciones preventivas deben ser apropiadas a los efectos de los problemas potenciales (pp. 13-16).

Una vez considerados los principales elementos que dan forma a la norma ISO 9001 se identifican sus principales beneficios en términos cualitativos y cuantitativos.

\section{BENEFICIOS DE LA NORMA ISO 9001}

\subsection{Beneficios cualitativos}

De acuerdo con Yáñez (2008), los principales beneficios que la norma ISO 9001 representa para una organización son los siguientes:

- Mejora continua de la calidad de los productos y servicios que ofrece.

- Mejora en la atención amable y oportuna a sus usuarios. 
- Transparencia en el desarrollo de procesos.

- Aseguramiento del cumplimiento de sus objetivos, en apego a leyes y normas vigentes.

- Reconocimiento de la importancia de sus procesos e interacciones.

- Integración del trabajo, en armonía y enfocada en procesos.

- Adquisición de insumos acorde con las necesidades.

- Delimitación de funciones del personal.

- Mayor satisfacción y mejor opinión del cliente.

- Aumento de la productividad y eficiencia.

- Reducción de costos.

- Mejor comunicación, moral y satisfacción en el trabajo.

- Una ventaja competitiva y un aumento en las oportunidades de ventas.

\subsection{Beneficios cuantitativos}

Los beneficios cuantitativos que el sistema de gestión de calidad puede otorgar a las empresas que se adscriben a las normas ISO 9000 están relacionados con la incidencia positiva en sus resultados financieros.

De acuerdo con la evaluación del impacto de los sistemas de gestión de calidad en la liquidez y rentabilidad de las empresas en una zona indus- trial, realizada por Fontalvo (2011), los beneficios financieros que los sistemas de gestión de calidad brindan a las empresas son positivos. Según este autor:
Los sistemas de Gestión de Calidad tienen una incidencia positiva en los indicadores de liquidez y rentabili- dad. Esta premisa resultante es co- herente con la teoría financiera, ya que si una empresa mejora todos sus procesos de gestión administrativa incrementa los indicadores antes mencionados, lo que se traduce en que la empresa tiene menos riesgo de entrar en falla ante situaciones financieramente difíciles en el corto plazo, ya que cuenta con una mejor capacidad de cumplir con sus obli- gaciones financieras, lo cual garan- tiza una mejor situación de la em- presa (p. 331).

A consideraciones similares llegan otros estudios, entre los que se cuenta el de Morelos, Fontalvo y Vergara (2013) respecto al impacto que tiene sobre las empresas certificarse en la norma ISO 9001, en particular, en sus indicadores de productividad y utilidad financiera. Los autores sostienen:

La certificación en calidad ISO 9001 de las empresas incide positivamente en los índices de productividad, razón utilidad bruta/valor agregado, y este último, a su vez, también incide positivamente, por la misma es- 
tructura financiera, en los resultados margen bruto y margen operacional, lo que permite inferir la importancia que tienen los procesos de certificación para el mejoramiento en la eficiencia productiva, creación de valor de productos y generación de riqueza (p. 107).

A partir de las conclusiones de los trabajos de múltiples investigadores, como los mencionados, es posible identificar que los sistemas de gestión de calidad mejoran los indicadores productivos y financieros de las empresas que operan en consideración de los mismos. De este modo, se puede inferir que estos sistemas, sobre todo las normas ISO 9000, representan beneficios para las organizaciones.

\section{CAMBIOS QUE PLANTEA LA NUEVA VERSIÓN DE LA NORMA ISO 9001:2015}

En el momento de elaborar este artículo, la norma ISO 9001: 2008 es objeto de una serie de modificaciones. La Organización Internacional de Normalización (ISO) está trabajando en la revisión de la ISO 9001, como se ha indicado, el estándar principal para el sistema de la gestión de la calidad. Se prevé que esta revisión esté lista para ser publicada hacia el mes de septiembre de 2015 .
Después de que la última revisión de la ISO 9001 versión 2008 trajera algunos pequeños cambios, la versión presentada por la ISO para discusión indica, en esta oportunidad, que habrá modificaciones cruciales en la que está prevista para finales del tercer trimestre de 2015.

\subsection{Enfoque basado en riesgos}

Un cambio significativo presente en la revisión 2015 tiene que ver con el enfoque basado en riesgos. De hecho, tener en cuenta los riesgos es un requisito en varias cláusulas de la norma, en particular, en el énfasis en procesos, liderazgo y planificación. La documentación del sistema debe ser adecuada a los riesgos existentes para la conformidad de productos o servicios y la satisfacción del cliente. Considerar estos riesgos habilita a la organización para abordar múltiples oportunidades. En el futuro, las organizaciones deberán usar herramientas y mecanismos de gestión de riesgos y de ahí derivar el desarrollo de mecanismos y acciones de mejora en el sistema de gestión (DNV GL, 2014).

La gestión del riesgo supone un diseño preventivo del sistema de gestión, con lo que desaparece el apartado específico de "acción preventiva". Se pide a las organizaciones que identifiquen el contexto en 
donde operan y localicen los riesgos y las oportunidades que deben tratarse. Aunque no se define con mucho detalle metodológico cómo documentar el requisito, sí debe ser la base para el diseño del sistema de gestión (European Quality Assurance [EQA], 2014).

\subsection{Del cliente a las partes interesadas}

Otro aspecto destacable es la ampliación del segmento específico al que tradicionalmente la norma ha dedicado sus esfuerzos. La idea, como señala Bustinza (2014), es que "además de comprender las necesidades de los clientes, la ISO 9001: 2015 requiere comprender las necesidades y expectativas de todas las partes interesadas" (p. 30).

La revisión realizada en 2015 trata de identificar y satisfacer las necesidades y expectativas no solo del grupo de clientes, sino de todos los grupos de interés (stakeholders) de la firma. Este replanteamiento conduce, además, "a considerar el entorno socioeconómico de la organización en la planificación del sistema de calidad" (Rey, 2013, p. 32).

\subsection{Enfoque en procesos}

A diferencia de lo que sucedía en las versiones precedentes de la norma, en la actual revisión el enfoque en procesos ya no es una recomendación y, en adelante, es una exigencia, un requerimiento a la organización (DNV GL, 2014).

\subsection{Información documentada}

Algunos elementos que eran considerados como evidencia, como una prueba clara y explícita de lo que soporta el diseño, la implementación, el desarrollo y la evolución del sistema de calidad, de naturaleza y denominación múltiples se integran en un único concepto. Así "con la intención de hacer el sistema de gestión más flexible y trazable, los actuales términos documentos, registros, procesos documentados, etc. serán reemplazados por el término genérico información documentada" (DNV GL, 2014, p. 2).

\subsection{Bienes y servicios}

Otro concepto que también se modifica, con el fin de ampliar el espectro de aplicación de la norma y de hacerla más adecuada a las realidades actuales del mercado, es el de "producto", el cual era utilizado de manera central en las anteriores versiones de la norma. A partir de la presente revisión, "en lugar de producto, se usará el término "bienes y servicios"'. Usando este término se pretende adaptar el 
estándar para mayor claridad a los proveedores de servicios" (DNV GL, 2014, p. 2).

\subsection{Gestión del conocimiento y gestión de competencias}

En adelante, se reconoce una mayor relevancia y visibilidad no solo a los tradicionales asuntos vinculados con el conocimiento explícito de la organización (documentación de procesos, etc.), sino también con cuestiones de naturaleza más bien tácita (Nonaka \& Takeuchi, 1995). De hecho, "la nueva versión 2015 da importancia a la gestión del conocimiento como activo intangible" (Bustinza, 2014, p. 30).

\subsection{Principios de gestión de calidad}

Como se mencionó, hasta la versión de 2008 la norma ISO 9001 se basó en ocho principios de gestión de calidad (figura 3). En la revisión realizada para su publicación en 2015, se ha llevado a cabo una modificación en este aspecto por la que se reducen esos principios de ocho a siete y se presentan en la figura 10 .

Figura 10. Siete principios de la gestión de calidad desde la versión 2015

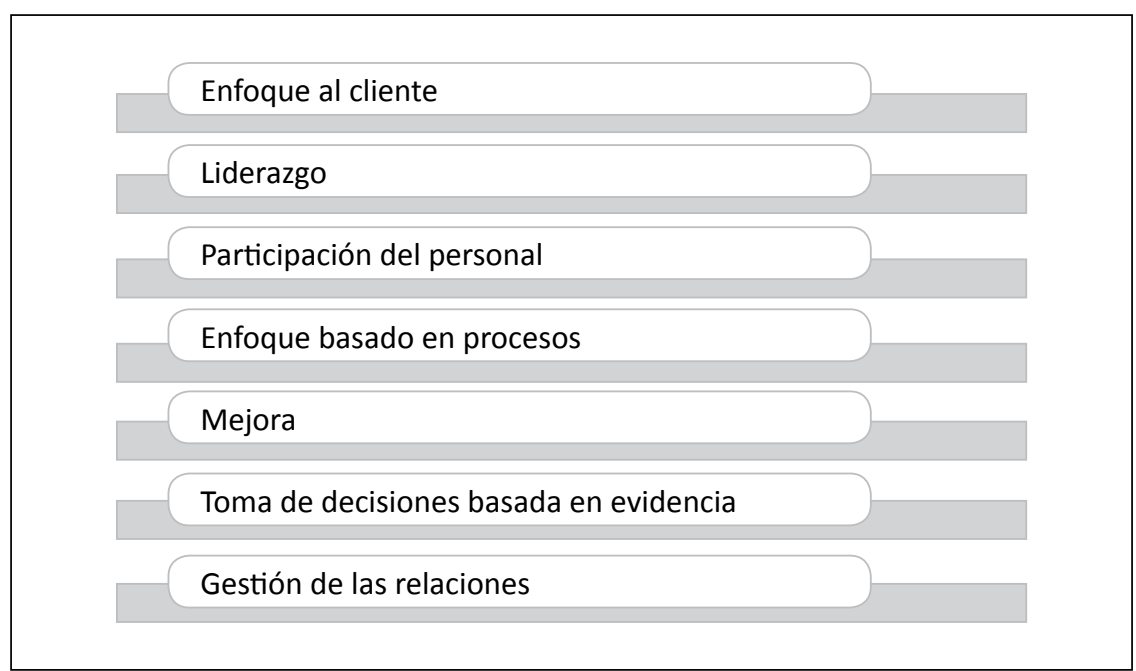

Fuente: elaboración propia con base en López (2013, p. 1).

Como se observa en la figura 10 , existen dos cambios básicos en la versión 2015. Por un lado, desapa- rece el cuarto principio, denominado "enfoque del sistema para la gestión" (figura 3), debido a que se 
considera que la interrelación, por sí misma, transforma la totalidad de los elementos en un sistema de gestión, lo que convierte a este principio en algo redundante. Por otro lado, el octavo principio, llamado "relaciones mutuamente beneficiosas con el proveedor" (figura 3) se transforma en el nuevo principio de "gestión de las relaciones", puesto que, como se indicó en la sección 4.2., la versión 2015 busca hacer un mayor énfasis en las partes interesadas (stakeholders) y cambiar la tradicional visión que consideraba solo a los clientes y proveedores.

\section{CONCLUSIONES}

En la presente investigación se han identificado, señaladoy definido, con sustento en los documentos fuente y en algunos trabajos relevantes propios del dominio de estudio, los beneficios cualitativos y cuantitativos que la norma ISO 9001 de gestión de calidad brinda a las empresas.

Entre los principales beneficios reconocidos por las organizaciones como resultado de la adecuación a esta norma está la mejora continua de la calidad de los productos y servicios. También se destacan el posible avance en la atención amable y oportuna de las necesidades y expectativas de los usuarios, la transparencia en el desarrollo de procesos, el mejor aseguramiento en el cumplimiento de los objetivos y la existencia de un mayor apego a las leyes y normas vigentes.

Otros beneficios significativos son el reconocimiento de la importancia de los procesos existentes y sus interacciones, la integración del trabajo en armonía y enfocado a los procesos, la adquisición de insumos de acuerdo con las necesidades, una más clara delimitación de las funciones del personal, la posibilidad de contar con mayor satisfacción y mejor opinión del cliente, el aumento de la productividad y la eficiencia, la reducción de costos, mejor comunicación, una más elevada moral y satisfacción en el trabajo, una eventual mayor ventaja competitiva y un incremento en las oportunidades de ventas.

Los beneficios financieros que proporciona la aplicación de la norma ISO 9001 a las empresas son tangibles. Existe una incidencia positiva de la ISO 9001 en indicadores esenciales del desempeño de las empresas. La mejora en los procesos de gestión administrativa, obtenida por la adecuación a la norma, constituye el soporte básico del mejoramiento visible en estos indicadores.

Con sustento en documentos fuente y en alguna literatura relevante, en 
este trabajo se identificaron y describieron los principales cambios que traerá para las empresas la versión 2015 de la norma ISO 9001. Los más destacados consisten, en primer lugar, en la presencia de un decidido enfoque basado en riesgos. En la nueva versión, el término "riesgo" comienza a formar parte de múltiples cláusulas de la norma, en particular, en el enfoque a procesos, el liderazgo y la planificación. De este modo, la documentación del sistema de calidad debe ser adecuada a los riesgos existentes para la conformidad de productos o servicios y la satisfacción del cliente.

En segundo lugar, se encuentra el cambio de un "enfoque en clientes" a uno en "partes interesadas". En este sentido, la versión ISO 9001:2015 se aproxima a los esquemas de calidad total (Pyzdek \& Keller, 2013), ya que requiere comprender las necesidades y expectativas de otras partes interesadas, además de los clientes. Es necesario tener en cuenta al entorno socioeconómico de la organización a la hora de pensar en la planeación del sistema de calidad.

Otro cambio que se destaca tiene que ver con los principios de gestión de calidad. En la nueva versión, desaparece el principio de "enfoque del sistema para la gestión", debido a que la interrelación, en sí misma, transforma todos los elementos en un sistema de gestión, lo cual hace que este sea redundante. Por otro lado, se modifica el principio de "relaciones mutuamente beneficiosas con el proveedor" y se habla del principio de "gestión de las relaciones, pues la versión 2015 pretende enfatizar en las partes interesadas, lo que cambia la visión que incluía solo a clientes y proveedores.

En la nueva versión se presta más atención a los procesos, productos y servicios y se otorga una mayor importancia a la gestión del conocimiento en tanto activo intangible, pero valioso. Asimismo, la gestión de los riesgos y la comprensión de los grupos de interés son dos asuntos esenciales para las empresas que deseen aplicar a esta prestigiosa certificación internacional.

Los cambios aquí estudiados han sido establecidos con base en los documentos fuente, en particular, en el borrador de la nueva versión de la norma ISO 9001, emitido por la Organización Internacional de Normalización (ISO). Estos se han decantado y presentado en este artículo con el fin de informar tanto a los académicos interesados como a las organizaciones que cuentan con la certificación ISO 9001:2008 y a aquellas que decidan certificarse en adelante, respecto a las modificaciones que 
tendrán que considerar las empresas interesadas en este sello.

\section{REFERENCIAS}

Alcalde San Miguel, P. (2008). Calidad. Madrid: Paraninfo.

Bustinza, S. (2014). La ISO 9001 y el camino a su nueva versión. Mercados \& Regiones, 2, 30. Recuperado de http://issuu.com/ mercadosyregiones/docs/mercados_regiones2

Centro de Desarrollo Industrial [CDI]. (2013). Empresas certificadas con ISO 9001 en el Perú. Recuperado de http://www.cdi. org.pe/asist_empcertISO9000040.htm

Comité Técnico ISO/TC 176. (2005). Norma internacional ISO 9000: sistema de gestión de la calidad. Fundamentos y vocabulario. Ginebra: International Organization for Standardization.

Comité Técnico ISO/TC 176 (2008). Norma internacional ISO 9001: sistema de gestión de la calidad. Requisitos. Ginebra: International Organization for Standardization.

Cuatrecasas, L. (2012). Gestión de la calidad total. Madrid: Díaz de Santos.

DNV GL (2014). Gestión de la calidad ISO 9001:2015. Recuperado de http://www.dnvba.com/es/Informacion- prensa/Documents/ ISO90012015.pdf
European Quality Assurance [EQA]. (2014) Novedades en la norma ISO 9001:2015. Madrid: European Quality Assurance.

Feigenbaum, A. (1983). Control total de la calidad (3a ed.). Nueva York: McGraw-Hill.

Fontalvo, T. (2011). Evaluación del impacto de los sistemas de gestión de calidad en la liquidez y rentabilidad de las empresas de la zona industrial de Mamonal. Revista Virtual Universidad Católica del Norte, 34, 314-334.

Hoyle, D. (2006). ISO 9000 Quality Systems Handbook (5a ed.). Oxford: Butterworth-Heinemann.

Instituto nacional de defensa de la competencia y de la protección de la propiedad intelectual [Indecopi]. (2012a). Perú solo tiene mil empresas con certificación de calidad. RPP Noticias. Recuperado de http://rpp.pe/economia/ economia/indecopi-peru-solotiene-mil-empresas-con-certificacion-de-calidad-noticia-532152

Instituto nacional de defensa de la competencia y de la protección de la propiedad intelectual [Indecopi]. (2012b). Unas $800 \mathrm{em}-$ presas cuentan con certificación de gestión de calidad. RPP Noticias. Recuperado de http://rpp. pe/economia/economia/unas800-empresas-cuentan-con-certificacion-de-gestion-de-calidadnoticia-525276 
Instituto Nacional de Estadística e Informática [INEI]. (2013). Estructura empresarial 2012.Lima: Depósito Legal Biblioteca de la Nación.

International Organization for Standardization [ISO]. (2013). ISO 9001:2015. Quality Management Systems. Requirements (Committee Draft). Ginebra: ISO.

López, L. (2013). Cambio en los principios de calidad para la norma ISO 9001:2015. Americantrustregister. Recuperado de http:// americantrustregister.blogspot. com.co/

Ministerio de Comercio Exterior y Turismo. (2014). Acuerdos comerciales del Perú. Recuperado de http://www.acuerdoscomerciales.gob.pe/

Morelos, J., Fontalvo, T. \& Vergara, J. (2013). Incidencia de la certificación ISO 9001 en los indicadores de productividad y utilidad financiera de empresas de la zona industrial de Mamonal en Cartagena. Estudios Gerenciales, 29(126), 99-109.

Nonaka, I. \& Takeuchi, H. (1995). The Knowledge-Creating Com- pany: How Japanese Companies Create the Dynamics of Innovation. Nueva York: Oxford University Press.

Oficina Internacional del Trabajo [OIT]. (2007). Informaciones generales sobre la Organización Internacional de Normalización. Recuperado de http://www.ilo. org/wcmsp5/groups/public/--ed_norm/---relconf/documents/ meetingdocument/wcms gb_298_15_5_add_es.pdf

Pyzdek, T. \& Keller, P. (eds.). (2013). The handbook for quality management: A complete guide to operational excellence (2a ed.). Nueva York: McGrawHill.

Rey. D. (2013). ¿Qué nos depara la futura ISO 9001:2015? Calidadnetwork. Recuperado de http:// www.calidadnetwork.com/numero4/files/assets/basic-html/ page $32 . h t m l$

Yáñez, C. (2008). Sistema de gestión de calidad en base a la norma ISO 9001. Internacional eventos. Recuperado de http://internacionaleventos.com/articulos/articuloiso.pdf 